\title{
LOS FUNDAMENTOS MENGERIANOS DE LA ESCUELA AUSTRIACA DE ECONOMÍA Y LA TEORÍA AUSTRIACA DEL CICLO ECONÓMICO: VÍNCULOS Y CONTROVERSIAS*
}

\author{
Mengerian foundations of the Austrian \\ School of Economics and the Austrian \\ Business Cycle Theory: linkages and \\ controversies
}

ANDRÁS TOTH**

Fecha de recepción: 12 de febrero de 2020

Fecha de aceptación: 8 de octubre de 2020

Resumen: La teoría austriaca del ciclo económico (ABCT), basada en las ideas de Böhm-Bawerk y Mises, se ha convertido en una de las ideas teóricas centrales de la Escuela Austriaca de Economía. La crisis de 2008 pareció dar la razón a la $\mathrm{ABCT}$. No obstante, el daño relativamente pequeño causado por la crisis y la expansión económica más larga jamás vista, refutaron las advertencias sobre la hiperinflación y el colapso de la civilización.

El objetivo de este artículo es explicar la causa del relativo éxito del sistema monetario basado en la creación de crédito mediante la reconstrucción del marco teórico original mengeriano combinado con las ideas misesianas.

En primer lugar, el artículo reconstruye los principales pilares de pensamiento de Carl Menger. Se analiza cómo el pensamiento post-Mengeriano se bifurcó en dos conceptos contradictorios en relación al papel del sistema de créditos; por una parte la teoría de Schumpeter y, por otra, la de Mises. El artículo muestra cómo Schumpeter distorsionó el pensamiento mengeriano original para explicar los ciclos económicos, mientras que Mises, bajo la influencia de Böhm-Bawerk,

\footnotetext{
* Me gustaría expresar mi gratitud a Pablo Yusta por su ayuda y apoyo y a los redactores que han aportado valiosas sugerencias.

** Senior research fellow, ELKH TK PTI, Director of Carl Menger Institute, Budapest, tothandhu@gmail.com
} 
descartó algunos supuestos clave de Menger; por ello la $A B C T$ refleja en menor grado los impactos del boom empresarial permitido por la expansión del crédito. En la discusión, propongo una reforma de $A B C T$, basada en la combinación del marco original mengeriano y las ideas misesianas. Esta reconceptualización de ABTC explica por qué no se ha producido una fase de hiperinflación a pesar de la expansión del sistema de créditos de las últimas décadas, y advierte de que el peligro real no es la hiperinflación sino el surgimiento de un gobierno omnipotente sobre la base de la expansión del sistema de créditos que allane el camino hacia la servidumbre.

Palabras clave: Teoría Austriaca del Ciclo Económico, Escuela Austriaca de Economía.

Clasificación JEL: A10, B13, B53, D50, E14, E32, E58, P16xí.

Abstract: The Austrian Business Cycle Theory (ABCT), based on the insights of Böhm-Bawerk and Mises, became one of the core theoretical insights of the Austrian School of Economics.

The 2008 crisis seemed to support the $A B C T$. Nonetheless, the relatively small harm caused by the crisis and the longest economic expansion ever seen refuted warnings about hyperinflation and civilizational collapse.

The aim of this paper is to explain the cause of the relative success of the credit expansion monetary system through the reconstruction of the original Mengerian theoretical framework combined with the Misesian insights.

First, the paper reconstructs Carl Menger's main tenets of thought. The paper discusses how the post-Mengerien development diverged into two conflicting concepts concerning the role of credit: the one conceptualized by Schumpeter and the one conceptualized by Mises. The paper shows how Schumpeter distorted the original Mengerian thought in order to explain business cycles, while Mises, under the influence of Böhm-Bawerk, discarded some of Menger's key assumptions making $A B C T$ less reflective of the impacts of the entrepreneurial boom allowed by credit expansion. In the discussion, I argue that the reformation of $A B C T$ based on the combination of the original Mengerian framework and the Misesian insights explains both the resilience and success of the credit based monetary system, but also warns that the real danger is the rise of an omnipotent government based on credit expansion that can pave the road to serfdom.

Keywords: Austrian Business Cycle Theory, Austrian School of Economics.

JEL Classification: A10, B13, B53, D50, E14, E32, E58, P16xí. 


\section{INTRODUCCIÓN}

Desde que el presidente Nixon suspendió la convertibilidad del dólar en oro en 1971, comenzó una expansión crediticia mundial. Esta expansión del crédito se convirtió en una explosión crediticia después de que Volcker lograra contener la inflación, lo que permitió el inicio del largo período de recorte en las tasas de interés por parte de los bancos centrales. La gran recesión de 2008 pareció dar la razón a la Teoría Austriaca del Ciclo Económico (TACE): los ciclos económicos son la consecuencia del crecimiento excesivo del crédito bancario debido a las tasas de interés artificialmente bajas establecidas por el tándem interconectado de bancos centrales y bancos comerciales. Una vez que la inflación provoca un aumento en la tasa de interés, el auge se convierte en quiebra y las malinversiones del período de bonanza causan pérdidas y bancarrotas. No obstante, la crisis ha sido gestionada con una nueva expansión crediticia y nuevos recortes en las tasas de interés. (ver: Tabla 1)

TABLE 1. US CREDIT EXPANSION. HTTPS://FRED.STLOUISFED.ORG/SERIES/TCMDO

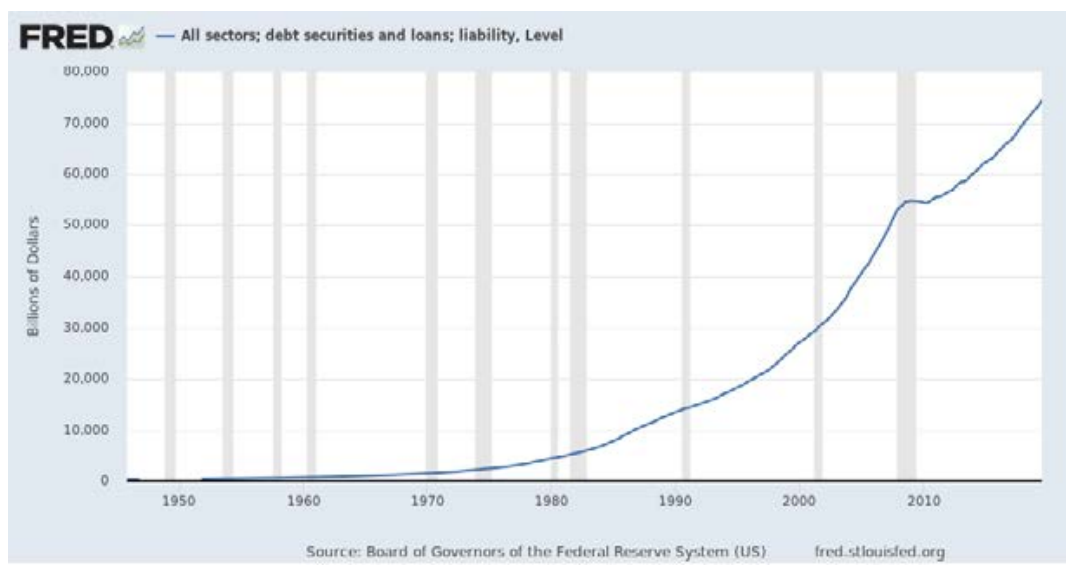

La recuperación relativamente rápida de la crisis a través de esas medidas expansivas hizo que los partidarios de la TACE 
fueran vistos como traficantes de miedo poco fiables, cuyas advertencias sobre la hiperinflación y el colapso civilizacional no estaban correctamente fundamentadas. En lugar de tratar de evitar la inflación, los bancos centrales trataban de perseguirla, pero sin éxito y no podían ni llegar a la inflación anual del $2 \%$, que era su objetivo. La opinión de los economistas del mainstream es que el pensamiento monetario austriaco se basa en el patrón oro y la TACE es una retrospectiva hacia un sistema inestable y fallido del siglo XIX. (Eichengreen and Flandrea 1985, Ahiakpor 2003, 112). Esta es la razón de que las advertencias y sugerencias austriacas no sean tomadas en cuenta (Block 2019). La falta de hiperinflación ha reforzado la opinión generalizada de que las ideas keynesianas-walrasianas han impulsado un aumento sin precedentes de la innovación y del bienestar, al mismo tiempo que han asegurado la estabilidad de la política democrática. A pesar de los peligros de la expansión del crédito, es cierto que el sistema monetario posterior al oro produce expansiones económicas cada vez más largas y, entre todas ellas, la más larga es la expansión actual que comenzó después de la última crisis crediticia en 2008.

El artículo argumenta que la TACE, que se basa en la obra de Mises, está en gran medida conformada por la rígida construcción lógica de capital e interés de Böhm-Bawerk, construcción que fue creada para refutar el marxismo. Por esta razón, carece del dinamismo reflexivo del marco mengeriano. Este dinamismo reflexivo mengeriano es más bien característico de la teoría del ciclo de auge y caída de Schumpeter, que influye fuertemente en las modernas teorías macro monetarias no austriacas. No obstante, Schumpeter también se desvió del marco teórico mengeriano y por eso, creó una teoría del ciclo rígida. Para solucionar el problema creado por esta rigidez interna, Schumpeter aboga por el intervencionismo estatal, una solución completamente ajena a Menger. Mises, por otro lado, siguió las pautas de Menger y rechazó el intervencionismo estatal más abiertamente, incluso el intervencionismo de los bancos centrales.

El objetivo de este artículo es reformular la TACE basándose en el marco teórico mengeriano original combinado con las ideas de Mises respecto al papel de los bancos centrales y del peligro del poder estatal omnipotente. Tal reformulación de la TACE 
permitiría explicar la causa del aparente éxito del sistema monetario moderno basado en expansiones crediticias utilizando las ideas austriacas, pero también podría resaltar el peligro a largo plazo del auge crediticio administrado por el estado.

La estructura del ensayo es la siguiente. Primero, el artículo reconstruye los principales postulados del pensamiento de Carl Menger. A continuación, el documento discutirá, en la sección II, cómo el desarrollo del pensamiento austriaco posterior a Menger divergió en dos conceptos contradictorios sobre el papel del crédito en la economía de mercado: uno conceptualizado por Joseph Schumpeter y otro por Ludwig von Mises. El artículo muestra, en la sección II.1, cómo Schumpeter tergiversó el pensamiento mengeriano original para explicar los ciclos económicos. En la sección II.2, el documento argumenta que Mises, bajo la influencia de Böhm-Bawerk, descartó algunos supuestos clave de Menger provocando que la TACE fuera menos reflexiva sobre los impactos positivos del auge empresarial surgido por la expansión crediticia. En el artículo, se defiende que el verdadero peligro es el crecimiento del gobierno omnipotente (Mises, 1944) a raíz de la expansión del crédito, que allana el camino hacia la servidumbre (Hayek, 1944).

I.

\section{EL ENFOQUE MENGERIANO}

El principal e influyente libro de Carl Menger, Principios de Economía (1871), tenía la intención de ser el primer libro de una serie completa de libros. Por lo tanto, sólo pretendía establecer las preguntas más fundamentales sobre la teoría económica. La teoría de Carl Menger, por lo tanto, se limitó a establecer los componentes básicos de una teoría causal de la economía y sirvió como base para un mayor desarrollo teórico en muchas áreas, incluida la teoría monetaria y la de los ciclos económicos de Schumpeter y Mises.

Menger tuvo como objetivo corregir la errónea teoría del valor de la economía clásica británica y refutar la negación de la existencia de leyes económicas por parte de la Escuela Histórica Alemana (Hayek 1976, Salerno 1999). Los Principios de Economía de Menger 
reconstruyeron la economía sobre una teoría del valor subjetivista a través de una estructura lógica de mecanismos causales entre los fenómenos económicos.

Menger argumentó que los mercados son la creación de microacciones espontáneas de los seres humanos que buscan la mejor manera de satisfacer sus necesidades económicas y que no fueron creados por fuerzas ciegas independientes de las necesidades o características humanas. Los mercados y las acciones humanas se rigen por las leyes de mercado que a su vez están determinadas por las necesidades humanas. Estas leyes se transmiten a través de mecanismos de causa y efecto.

La principal diferencia con las leyes naturales es que éstas se aplican sobre los objetos inanimados, los meros ejecutores. Los seres humanos piensan, actúan, se equivocan, planean, cada uno con habilidades individuales, valoraciones y objetivos subjetivos. En consecuencia, las leyes del mercado establecen las condiciones para la acción humana y no determinan los movimientos exactos de cada persona en cada situación. Las leyes del mercado favorecen a la acción humana, como interpretó Mises, a través del mecanismo de causa y efecto, que castiga o recompensa el comportamiento humano a través del mecanismo de pérdidas o ganancias. Estos mecanismos de causa y efecto ayudan a las personas que actúan a encontrar la mejor solución posible para satisfacer sus necesidades.

Menger era consciente del hecho de que las instituciones políticas son tan poderosas que podrían distorsionar el mecanismo de causa y efecto de las leyes de mercado. Por esta razón, Menger observa y deduce las leyes del mundo imaginario y aislado de la "acción puramente económica",1 aislada del mundo político. Las leyes que buscaba descubrir no eran los diseños de un planificador omnipotente, sino las consecuencias de innumerables acciones no planificadas que surgen en la vida económica a través de la acción humana.

La teoría del valor subjetivista de Menger se basa en su concepción del ser humano: "el hombre mismo es el principio y el fin de

\footnotetext{
${ }^{1}$ Expresión de Böhm-Bawerk, citada en las memorias de Mises (2009, p. 44.).
} 
toda economía" (citado por Yagi 1993). El actor económico para Menger es la persona que economiza en el contexto de inseguridad y escasez. La palabra economizar es algo engañosa, ya que se suele asociar con la prudencia y ese concepto no describe la idea de Menger sobre los seres humanos. En los primeros capítulos de Principios, donde analizó las causas del progreso de la civilización, Menger conceptualizó una idea más amplia de persona economizadora. En este concepto, persona economizadora significa una persona emprendedora. Una persona capaz de descubrir nuevas relaciones causales, capaz de llevar a cabo estos descubrimientos, ampliar sus conocimientos y planificar en el futuro, no sólo que sea capaz de manejar con prudencia los recursos disponibles.

La persona emprendedora (economizadora) mengeriana tiene dos rasgos inherentes entrelazados: la capacidad de aumentar el conocimiento y de tener continuamente necesidades cada vez mayores. Según Menger, el progreso verdadero y duradero se logrará cuando las personas ya no consideren los objetos como acontecimientos no relacionados, sino que intenten descubrir sus conexiones causales y las leyes a las que están sujetos. Para Menger, esta capacidad humana inherente para aumentar el conocimiento es el verdadero motor del progreso de la civilización.

La expansión del conocimiento está entrelazada con la inherente capacidad humana de tener continuamente necesidades cada vez mayores. El conocimiento expande las necesidades, las nuevas necesidades obligan a los humanos a encontrar nuevas soluciones (1871, 77). Tanto las necesidades humanas, como el conocimiento, son capaces de un crecimiento infinito. Para Menger, la causa de que siempre exista escasez es que "el concepto de infinito es aplicable sólo al progreso ilimitado en el desarrollo de las necesidades humanas, pero no a las cantidades de bienes necesarios para la satisfacción de estas necesidades durante un período de tiempo dado" $(1871,83)$. Es decir, las necesidades potencialmente infinitas siempre están por delante de la oferta existente de bienes, y por lo tanto esta escasez inherente obliga a las personas a obtener nuevos conocimientos para satisfacer sus necesidades urgentes.

Estos componentes internos que provocan unas necesidades cada vez mayores y la expansión del conocimiento provocan un mundo económico dinámico. El dinamismo del mundo económico 
significa que la economía mengeriana es una economía del desequilibrio, impulsada principalmente por los factores humanos endógenos de la expansión del conocimiento y las necesidades. En este mundo dinámico, sólo hay equilibrios parciales y momentáneos, cuando dos actores acuerdan un intercambio y, por lo tanto, satisfacen sus necesidades por un tiempo $(1871,188)$.

La consecuencia de la constante expansión del conocimiento humano es la creciente variedad y complejidad de los bienes necesarios para satisfacer esas necesidades que también se encuentran en constante expansión. Esto requiere un proceso de producción cada vez más largos para generar productos más complejos y requiere también de una planificación cada vez más larga por parte de las personas emprendedoras.

En la cadena causal mengeriana, la aparición de mercados es consecuencia de la expansión del conocimiento. Los mercados surgen cuando los hogares aislados y las comunidades humanas cerradas no pueden satisfacer todas sus necesidades en el marco de una economía doméstica o comunitaria cerrada y autosuficiente. El intercambio en los mercados permite a las personas intercambiar los bienes que valoran menos por otros que valoran más con otras personas que, a su vez, están dispuestas a intercambiarlos pues valoran menos el bien que ellos producen que el bien que producen las otras personas. Además, los bienes al ser cada vez más complejos requieren el suministro de bienes intermedios, como bienes de producción, materias primas y productos intermedios (bienes de orden superior en el lenguaje mengeriano) para poder producir bienes de consumo (bienes de primer orden en el lenguaje mengeriano). Esto lleva a la aparición de cadenas de producción complejas e interdependientes en los mercados en evolución, donde el intercambio de bienes de primer orden se entremezcla con el intercambio de bienes de orden superior, con el objetivo de producir bienes complejos de primer orden que puedan satisfacer las crecientes necesidades humanas.

El desarrollo de cadenas de producción largas y complejas aumenta la magnitud de las posibles disrupciones inesperadas debido a nuevos conocimientos (innovación), cambio de gustos o de tendencias, o cualquier evento externo (como una guerra que afecte a uno o más productores). Este tipo de crisis, sin embargo, es sólo parcial y 
limitada en el sistema mengeriano. En caso de crisis, el mercado ayuda a las personas emprendedoras a reconfigurar su capital, o a obtenerlo a través de crédito y así, poner en marcha nuevas ideas o buscar nuevas posibilidades de producción. De esta manera, el mercado facilita a los seres humanos la posibilidad de desarrollar una nueva actividad económica a través de la ampliación del conocimiento, la innovación y la adaptación a los nuevos desafíos

Esta creciente complejidad de las cadenas de producción, junto con la necesidad de más y más bienes de orden superior para producir bienes de primer orden y la ampliación del plazo de producción requieren un capital cada vez mayor para poder sostener la estructura productiva, mientras que los bienes de orden superior se transforman en bienes de primer orden que son capaces de satisfacer las necesidades de consumidores finales. Para Menger, el sello distintivo del progreso de la civilización es la creciente cantidad de capital disponible para las personas emprendedoras.

Menger hizo una distinción entre empresario y emprendedor en función de su relación diferente con el capital. Como hemos visto antes, Menger ha conceptualizado al hombre emprendedor. Ser emprendedor es una cualidad inherente al ser humano. Pero sólo algunos llegan a ser empresarios. Los empresarios son aquellas personas emprendedoras, que tienen el control sobre el capital. Menger argumentó que la escasez de capital limita la función empresarial, y el crédito aumenta la cantidad de capital para desarrollarla $(1871,172)$.

El concepto de capital de Menger en los Principios (1871) cubre el conjunto de bienes de orden superior (bienes de capital), dinero o crédito gestionados por un empresario. Menger argumentó que el dinero no es capital por definición, pero que tanto el dinero como el crédito sirven a la persona emprendedora para obtener el control sobre los bienes de capital. Menger enfatizó aún más la importancia del dinero como capital en su artículo de 1888. En este artículo, argumentó que el capital es dinero y es el valor monetario de los bienes de capital (Braun, 2013). El dinero (y el crédito) es capital "libre" o capital en sentido más amplio. El capital libre una vez invertido se convierte en un capital "específico" o capital en sentido más restringido. El concepto más restringido de capital es el conjunto específico de bienes de orden superior adquiridos o 
poseídos por el empresario capitalista con el propósito de transformarlos en un bien de orden inferior particular con la expectativa de obtener ganancias.

Menger argumentó que los empresarios invierten su capital libre en bienes de capital específicos porque el capital tiene productividad. Hasta cierto punto, la productividad del capital es un término engañoso en el caso de Menger. En el contexto de su obra deja claro que, para él, el capital no tiene una calidad mecanicista de producir más valor que el propio desembolso de capital original. La fuente de la productividad del capital para él es la de nuevo conocimiento de una persona emprendedora que emplea el capital, y que espera obtener ganancias invirtiendo ese capital. Por lo tanto, la productividad del capital es la expectativa subjetiva de una persona emprendedora de obtener ganancias invirtiendo su capital (o pidiéndolo prestado) para adquirir bienes específicos de orden superior para transformarlos en bienes de orden inferior.

En el marco mengeriano, el interés es el precio que paga el empresario al propietario del capital por hacer uso de ellos. El capital prestado permite al empresario adquirir un conjunto de bienes de orden superior para transformarlos en bienes de orden inferior. El interés es la compensación pagada por el empresario al dueño de los bienes de capital, que no quiere usar su capital como empresario. Fekete (2007), explicando la idea mengeriana, argumentó que para el empresario este intercambio es rentable, porque espera ganancias económicas por encima de los costes de los desembolsos de capital. Para la persona propietaria del capital, este intercambio también es rentable, porque genera ingresos a través de su riqueza y tiene la capacidad de garantizar la satisfacción de sus necesidades sin consumir su riqueza o de simplemente obtener mayores ingresos de los que generaría al invertir él mismo su riqueza como capital. Por lo tanto, el interés de los préstamos se basa en las expectativas subjetivas tanto del prestamista como del prestatario, y según Menger, no es correcto el concepto de Senior, quien pensaba que el interés es el de descuento sobre el bien futuro a pesar de la similitud superficial. ${ }^{2}$

2 La concepción mengeriana del interés está notablemente influenciada por Adam Smith: "Casi todos los préstamos se hacen con dinero...Pero lo que el prestatario 
En el marco mengeriano, la fuente del beneficio se encuentra al llegar a una posición de monopolio por el empresario o al competir con la posición de monopolio de otro empresario. Al analizar la formación de precios monopolísticos, Menger distingue dos tipos de monopolio:

1) El monopolio estrecho es cuando ciertos empresarios están protegidos por el estado o la comunidad de la competencia de otros empresarios.

2) El monopolio amplio o monopolio de la condición real es una situación en la que los empresarios, como resultado de sus propiedades, o debido a sus talentos o circunstancias especiales, pueden comercializar bienes que es física o económicamente imposible que puedan ser suministrados competitivamente por otras personas emprendedoras.

En ambos casos, el empresario que disfruta de una posición de monopolio podría cosechar ganancias monopolísticas. La diferencia entre los dos tipos es la naturaleza del monopolio y la falta o posibilidad de competencia.

En el caso de monopolio limitado, no hay espacio para la competencia debido a la regulación. En el caso de monopolio de la condición real, el espacio para la competencia está abierto: la competencia en este caso invita a desafiar el monopolio adquirido por un empresario.

En el caso de monopolio de la condición real, la fuente de ganancias es el nuevo conocimiento o las circunstancias especiales del emprendedor, que resultan en un monopolio real y que permiten obtener ganancias monopolísticas. A medida que la competencia se abra y los demás seres humanos emprendedores comiencen a producir el mismo bien o uno parecido, estos emprendedores que ingresan más tarde en la producción podrían esperar ganancias por encima de sus costes al competir con las ganancias del

realmente quiere, y lo que el prestador realmente proporciona, no es dinero, sino el valor monetario, o los bienes que se pueden adquirir... Mediante el préstamo, el prestamista, por así decirlo, cede al prestatario su derecho a cierta parte de la producción anual de terreno y de la mano de obra del país, para ser controlado según el criterio del prestatario." (1776, 37) 
monopolio actual hasta que los competidores no puedan satisfacer todas las necesidades posibles. Es decir, hasta que todos los productores alcancen el precio más bajo posible, lo que al menos asegura la tasa de ganancia mínima. Este es el nivel de precios que Adam Smith llamó precio natural.

Por lo tanto, en el marco mengeriano, la competencia en el mercado significa competir con el monopolio actual de un emprendedor innovador. La competencia fuerza la reducción de precios y lidera la producción a gran escala para satisfacer todas las necesidades posibles al precio más bajo posible, garantizando unas ganancias mínimas. Cuanto mayor sea la competencia, menos posible es que un emprendedor permanezca en el negocio sin hacer que la producción sea cada vez más eficiente. Por lo tanto, la competencia también reduce el desperdicio de recursos productivos para que sea lo más pequeño posible. Este concepto de competencia permitió a Menger descartar la teoría de Senior sobre la formación de capital basada en el ahorro: Menger argumentó que los ahorros (aumento de capital) provienen de la innovación y la eliminación de procesos improductivos durante la producción competitiva.

Para obtener nuevas oportunidades de ganancias, la competencia insta a los empresarios a recurrir a su capacidad humana inherente de descubrir nuevos conocimientos e inventar nuevos métodos de producción más baratos y efectivos, o un producto ligeramente modificado o sustancialmente modificado o incluso sacar un nuevo producto. Este impulso constante de innovación, de ampliación del conocimiento para obtener ganancias es lo que impulsa el avance de la civilización y asegura la mejor satisfacción posible de las necesidades de los clientes.

En el marco de valoración subjetiva de Menger, en última instancia, los clientes deciden si valoran o no la innovación y el esfuerzo de trabajo de los emprendedores a través de su decisión de comprar el bien ofrecido. Por lo tanto, las expectativas de ganancias de los empresarios sólo podrán hacerse realidad si son capaces de producir un bien de orden inferior que satisfaga las necesidades de los clientes.

En resumen, Menger tenía una visión optimista sobre el desempeño de los mercados. Pensaba que los mercados son instituciones 
formadas a través de la acción humana natural al tratar de lograr la mejor satisfacción posible de sus necesidades. Vio en los mercados no sólo instituciones de intercambio de bienes, sino también un lugar donde podrían surgir cadenas de producción al combinarse varios actores con el objetivo de producir un bien de orden inferior a partir de una combinación concreta de bienes de orden superior. Los mercados también se convierten en el lugar para asegurar capital para el futuro a través de préstamos, para los empresarios que no son dueños de bienes de capital. Así, el crédito facilita que las personas emprendedoras puedan ser empresarios y poner en marcha sus ideas innovadoras. El mercado de crédito es, por tanto, el mercado para intercambiar la riqueza de uno en capital para un empresario, donde el interés es el precio pagado por el uso temporal del capital por parte del empresario.

Menger conceptualizó un mundo en desarrollo progresivo, en el que el conocimiento, las necesidades, la división del trabajo, el capital disponible para la producción, están en constante crecimiento, donde el equilibrio es sólo un breve fenómeno temporal y limitado. En este contexto, el desequilibrio propio de las crisis también es temporal y tiene un efecto limitado. Los mercados ofrecen la oportunidad de pensar y planificar a los seres humanos para decidir sus planes y reinvertir su capital en una nueva producción innovadora y así poder superar la situación de crisis. Por lo tanto, en el mundo sin estado mengeriano analizado en los Principios de Economía no existe una causa para los ciclos recurrentes generalizados de auge y caída, ni tampoco existe una razón para que se produzca una crisis sistemática importante que amenace el colapso civilizacional como la Gran Depresión de 1929-1933.

La extensión de crédito, que luego se convierte en la causa principal de las explicaciones de la crisis austriaca, es un fenómeno benevolente para Menger. En principio, el crédito crea más oportunidades para que las personas emprendedoras se conviertan en verdaderos empresarios. Los empresarios utilizan bienes de capital para producir bienes de menor orden y obtener ganancias a través de una mayor satisfacción de las necesidades de sus consumidores. En cuanto a los efectos negativos de las creaciones de crédito en cuestión, sabemos por las conferencias que Menger dio al Príncipe Heredero Rudolf, que estaba en contra de la 
creación de dinero fiduciario por parte del gobierno, y pensó que Austria debería volver al patrón de dinero mercancía y los bancos deberían poder canjear sus billetes por oro en todo momento, si los propietarios de los billetes lo desean (Menger 1994).

Menger dejó un legado, presentó una nueva base teórica para la economía. Dejó a las próximas generaciones de economistas un marco teórico para investigar el impacto de la creación de crédito en el progreso de la civilización y su conexión causal con los ciclos de auge y recesión.

II. EL DESARROLLO POST-MENGERIANO. TEORÍAS DEL CICLO ECONÓMICO: SCHUMPETER VS. MISES.

La siguiente generación de economistas dentro de la teoría del valor subjetivo mengeriano no sólo ha refinado y enriquecido la herencia mengeriana, aplicando sus ideas a otras áreas, sino que ha modificado su estructura lógica hacia nuevas direcciones, lo que significa una desviación de la estructura lógica mengeriana.

Friedrich von Wieser abandonó el mundo económico puro (sin Estado) de Menger y se involucró en los análisis del entorno social (Streissler 1988, Arena y Gloria Palermo 2001). Wieser argumentó que una economía simple imaginaria, la economía pura mengeriana, sólo podía analizar al individuo aislado e idealizado (Wieser, 1893). Wieser, después de investigar primero los modelos simplificados, incluirá también "los poderes sociales que gobiernan en gran medida los destinos del hombre" (Wieser, 1927, 207).

Wieser también consideraba el intervencionismo estatal como una acción benevolente para salvaguardar la sociedad. En Economía Social, argumentó (1927, p.414) que para contrarrestar la "tiranía" y el "aplastante despotismo" del poder capitalista se requiere la acción del Estado, que está en armonía con "el espíritu social de la economía" para garantizar el mayor beneficio social posible.

La otra diferencia importante para nuestros análisis es que Wieser, bajo la influencia de Walras, estudió la economía en equilibrio. Wieser también había abandonado el concepto mengeriano de la importancia del emprendedor. Al contrario de Menger, pensó que 
el avance de la civilización se debe a grandes personas, reyes y legisladores; El Übermenschen de Nietzsche (Anderson, 2009: 77) y las masas sólo son capaces de actuar a través de un líder que puede unir a las multitudes en una unidad activa (Wieser, 1927: 319-328). En el marco wieseriano, el empresario es un gran capitalista que representa la superioridad personal, lo que en la era de las grandes empresas le otorga un grado de poder que se traduce en la supremacía capitalista sobre las masas. Todos, excepto el héroe emprendedor, se aferrarán lo más posible a los métodos económicos tradicionales (Anderson, 2009: 73). En lo que respecta a las opiniones monetarias de Wieser, siguió a la Escuela Bancaria (Hulsman, 226).

A diferencia de Wieser, el marco de investigación de BöhmBawerk se mantuvo dentro del contexto económico puro de Menger.

Böhm-Bawerk argumentó en contra de la errónea concepción marxista del interés, tanto moral como teóricamente, en su brillante libro Teoría Positiva del Capital, en el que empleó un concepto diferente de capital y tasa de interés al de Menger. Para mostrar de una manera más detallada el papel del interés en el proceso de producción, empleó un concepto limitado de capital que consiste únicamente en bienes de producción, un concepto de capital más limitado que el de Menger. También desarrolló el concepto de tiempo (agio) como causa del interés durante el proceso de intercambio de bienes presentes por bienes futuros en una economía de equilibrio. A pesar de que admitió que, en un entorno dinámico competitivo, la lógica de oferta y demanda de Menger también tiene un impacto en la tasa de interés, descartó la teoría de la tasa de interés de Menger. Menger estaba preocupado por el trabajo teórico de Böhm-Bawerk, y según Schumpeter (1954, p. 847) pensó que "la teoría del capital e interés de Böhm-Bawerk es uno de los errores más grandes jamás cometidos". Menger escribió su artículo de 1888, en el que enfatizaba la importancia del dinero como capital, para refutar el concepto limitado de Böhm Bawerk (Braun, 2013). A pesar de las contra-argumentaciones de Menger, el concepto de capital y tasa de interés de Böhm-Bawerk tuvieron un papel más importante en el desarrollo posterior del pensamiento económico austriaco, influyendo en Wicksell, Mises y Hayek. 
Böhm-Bawerk también desarrolló el concepto del roundaboutness de la producción y argumenta que a través del roundaboutness se podría medir la intensidad de capital de la producción, concepto que también influyó en el desarrollo de la TACE.

Fue el sueco Knut Wicksell, el primer pensador dentro de la tradición mengeriana-böhm-bawerkiana, quien teorizó sobre el papel del sistema monetario compuesto por bancos de reserva fraccionaria. Sostuvo que en un sistema de bancos con reserva fraccionaria el crédito se genera a partir de una oferta de ahorro dada (Wicksell 1906). Wicksell argumentó que los bancos mismos crean el dinero requerido por los prestatarios, y la demanda de capital en gran medida crea su propia oferta. En el mundo de la banca de reserva fraccionaria, el vínculo de conexión mengeriano entre intereses y ganancias ya no tiene sentido. En cambio, la tasa de interés afecta los precios y éstos, afectan a las ganancias. Cuando el interés es bajo en proporción a la tasa de ganancia existente, se produce inflación. El proceso inverso tiene lugar cuando la tasa de interés es demasiado alta en proporción al beneficio promedio del capital. Este movimiento de precios es clave en el marco de Wicksell, pues interconecta los cambios en las tasas de interés no sólo con los cambios en los precios, sino también con el concepto Böhm-Bawerkiano del roundaboutness de la estructura de producción. Las tasas de interés naturales generan una estructura de producción de equilibrio a la luz de las preferencias temporales (ahorro) y los rendimientos de la creación de bienes de capital. Si la tasa de préstamo es más baja que la tasa natural, causa el alargamiento de la estructura de producción, mientras que un aumento conduciría a un roundaboutness de producción más corto. Si hay una diferencia entre las tasas de interés naturales y de préstamo, esto causa expansión o contracción en el tiempo de producción, y ese proceso perturba el equilibrio. Por lo tanto, en el marco de Wicksell, el tipo de interés de los préstamos es el precio clave en la estructura de precios y regula el roundaboutness de la producción. Wicksell (1907) argumentó que los bancos nacionales podrían ponerse como objetivo la tasa natural y así lograr el equilibrio al mantener la estabilidad de los precios a través de su política de ajuste de la tasa de interés. Por esta razón abogó por un sistema monetario fiduciario dirigido por bancos nacionales competidores. 
En la primera década del siglo XX, Joseph Schumpeter y Ludwig von Mises, los miembros más brillantes de la siguiente generación de jóvenes economistas entrenados en la tradición mengeriana por Böhm Bawerk y Wieser, e influenciados por las teorías monetarias de Wicksell, asumieron la tarea de aventurarse en un área no teorizada por Menger: establecer el vínculo causal entre la creación de crédito por parte del sistema bancario y los ciclos de auge y caída, y qué papel juega o debe jugar el estado para evitar o disminuir el impacto de los ciclos económicos. Llegaron a conclusiones diferentes sobre el papel del crédito y ofrecieron soluciones distintas sobre cómo disminuir el impacto destructivo de la crisis crediticia. Sus percepciones teóricas divergentes y conflictivas, lo que adoptan del legado de Menger, lo que rechazan y cómo crearon una explicación sobre el papel del crédito es la clave para comprender el fracaso relativo y la verdad a largo plazo de la TACE. ${ }^{3}$

\section{EL DESARROLLO POST-MENGERIANO. TEORÍAS DEL CICLO ECONÓMICO: SCHUMPETER vs. MISES.}

A pesar de la posición ambigua de Schumpeter en la escuela austriaca ${ }^{4}$, su teoría se basa en la tradición mengeriana en temas clave:

1) Schumpeter conceptualizó la economía de mercado como un mecanismo dinámico de cambio económico (1943, p. 83).

2) La innovación de los emprendedores es el motor dinámico del progreso y el avance. La innovación es un concepto similar a la ampliación del conocimiento mengeriana.

${ }^{3}$ Ver también: McCaffrey (2013) sobre las diferencias entre Schumpeter and Mises.

4 Schumpeter tenía una relación ambivalente con la Escuela Austriaca (Salerno 1999b, Festre 2002). Schumpeter no es considerado parte de la Escuela Austriaca (Haberler 1951. p.29, Hayek 1968, 461.) Mises no le incluía entre los Austriacas ya que siguió a Wieser y a Walras, y no a Menger ni a Böhm Bawerk (Mises 2009, 28). Otros argumentan que Schumpeter es parte de la tradición Austriaca (Streissler 1983, 356-64, Kirzner 1990: 244). 
3) La innovación no es sólo invención, sino que puede incluir la introducción de un nuevo bien, un nuevo método de producción, el descubrimiento de nuevos mercados, nuevos productos de un orden superior, o una nueva organización de producción e industria $(1939,276)$; un concepto cercano a la posición de monopolio real de Menger.

4) Para Schumpeter, el capital es el saldo de dinero disponible para el empresario. El dinero (crédito) permite al emprendedor adquirir bienes de capital para embarcarse en la producción con expectativas de ganancias. El concepto de capital de Schumpeter es un concepto cercano al formulado por Menger en 1888. Schumpeter pone aún más énfasis en el papel del capital monetario que Menger. Para él, el capital monetario es un agente distinto en comparación con el capital real (bienes de capital), que se encuentra entre los empresarios y los bienes de capital. El aumento y la disminución del capital monetario no es lo mismo que el aumento y la disminución de commodities o cualquier clase particular de commodities $(1939,130)$.

5) La acumulación de capital se debe al proceso de producción innovador y de reducción de costes, que crea un capital más amplio para la producción futura, y no un aumento en el ahorro.

6) Los emprendedores están pagando intereses porque esperan obtener ganancias del uso del capital prestado (1939, 125).

7) La innovación es la fuente de ganancias (1939, p.85). La oportunidad de obtener ganancias es el motor del desarrollo, ya que moviliza a personas innovadoras para convertirse en emprendedores.

8) El crédito crea oportunidades para que los empresarios lleven a cabo su idea innovadora.

No obstante, el punto de partida de Schumpeter es diferente que para Menger. Para Schumpeter, el punto de partida es el equilibrio walrasiano/wieseriano de economía circular. En esta economía circular hay reacciones a eventos externos, hay progreso, aumento de la población y del capital, pero no hay "desarrollo, que 
consiste principalmente en emplear los recursos existentes de una manera diferente, en hacer cosas nuevas con ellos" (Schumpeter 1943, p. 65). El papel del dinero en esta economía circular es limitado: el dinero cumple el propósito de la unidad de cuenta y facilita la circulación de mercancías dentro de la economía (Festre, 2002). ${ }^{5}$ Para Schumpeter, a efectos prácticos, el Capital y el Interés son conceptos que tienen relevancia cuando de repente unos agentes con capacidad de liderazgo ponen en marcha un proceso de innovación y de desarrollo económico. No forman parte de la acción humana o de la actividad económica en sí, algo que aleja a Schumpeter del paradigma Mengeriano. El interés, para Schumpeter, de hecho es un fenómeno estrictamente del desarrollo.

El objetivo de Schumpeter era explicar cómo la economía comercial circular walrasiana/wieseriana se convierte en una dinámica capitalista, del tipo que fue analizado por Menger, y qué causa los ciclos de auge y caída de la economía capitalista.

Para explicar este fenómeno de dinamismo a través de crisis, Schumpeter se desvía del marco mengeriano en cuanto al perfil psicológico de los actores económicos y empresarios. Menger consideró que la ampliación del conocimiento es una habilidad humana inherente. El concepto schumpeteriano de actores económicos estaba basado en el concepto wieseriano de diferencia entre masas y de superhéroe emprendedor. En opinión de Schumpeter, el equilibrio inicial se debe a que la mayoría de las personas son simples seguidores de costumbres y prácticas establecidas (1934, 214). El papel de los superhéroes, de los emprendedores innovadores, es el de movilizar la economía circular. El emprendedor schumpeteriano, al contrario de las masas, tiene intuición, libertad mental, capacidad para aprovechar las oportunidades inmediatas (1934, p.314). El emprendedor innovador schumpeteriano es un superhumano con el objetivo de fundar "un reino privado, tener la voluntad de conquistar, la alegría de crear" (Schumpeter 1934, p.331). La clave de su éxito es su capacidad para aprovechar las oportunidades inmediatas. Sus decisiones deben tomarse "sin

${ }^{5}$ El concepto de dinero de la economía circular fue influenciado por la "economía de efectivo puro" de Wicksell (Ver: Wicksell 1898/1965, 51-58). 
resolver todos los detalles", se basan en su capacidad predictiva (Schumpeter, 1934, p.314).

Hay más rigideces en su concepto de empresario. Primero, distingue entre dos tipos de empresarios: entre empresarios heroicos y empresarios miméticos. Los empresarios heroicos son aquellos que revolucionan la producción de forma innovadora y, por lo tanto, crean una situación de cosecha de beneficios para ellos, similar a la del monopolio real de Menger. Los empresarios heroicos son pocos en número. Los empresarios miméticos son aquellos que copian la idea innovadora del empresario heroico y entran en la esfera de la producción con el mismo producto o uno similar con el fin de obtener el resultado de beneficio monopolístico del monopolio real, utilizando el lenguaje de Menger, en el contexto de la competencia. La conceptualización de dos clases de empresarios fue ciertamente influenciada por el concepto mengeriano de empresario real que creaba el monopolio y, más tarde, empresarios que competían por el beneficio monopolístico del empresario innovador original. Pero, Schumpeter emplea un concepto muy rígido de empresario innovador al limitar la capacidad innovadora de los empresarios innovadores a un solo acto y declarar que una vez que tienen éxito, pierden su capacidad innovadora y se convierten en meros gerentes de sus empresas, lo que significa, por lo tanto, que pierden su ventaja innovadora. En el caso de los empresarios miméticos, carecen de capacidad innovadora más allá de copiar la idea innovadora del empresario heroico.

Esta mayor rigidez aparece también en el concepto schumpeteriano de que los empresarios emergen en oleadas, y una vez que una ola (generación) de empresarios tiene éxito, pierden su ventaja innovadora, y sólo una próxima generación de empresarios podría inducir una nueva ola de innovación.

Estas rigideces son esenciales para Schumpeter, ya que crea su ciclo de auge y caída en base a estos rasgos psicológicos. ${ }^{6}$

Otro elemento clave en el ciclo de auge y caída de Schumpeter, es que los empresarios están actuando en una situación de "aislamiento con los medios de producción", no tienen ahorros ni capital

\footnotetext{
${ }^{6}$ Ver también: Mises $(1981,71)$ y Rothbard $(1963,69)$ sobre psicologización y rigidez del modelo psicológico schumpeteriano.
} 
propio (1934, 70 y 160), por lo que dependen del crédito. Aquí entra en juego la creación de crédito por parte del sistema bancario.

El éxito de los empresarios depende de que puedan obtener crédito de los bancos. Los bancos crean créditos "anormales" de la nada (1934, p. 102). El crédito, que desempeña el mismo papel que el dinero, permite a los empresarios implementar sus ideas innovadoras (Schumpeter 1934, p. 53). El crédito otorga poder de compra a los empresarios para poder ofertar por bienes de mayor orden utilizados por empresas en la economía circular. Por esta razón, el crédito es el "perturbador" de la economía circular, y no un "condicionante neutral de distribución" pasivo, un "velo" como fue conceptualizado por Böhm Bawerk (Schumpeter 1954, p. 10881089). El crédito hace posible la ruptura del equilibrio walrasiano de la economía circular de empresarios innovadores. En un sentido más amplio, la creación de crédito por parte de los bancos es el factor causal del surgimiento del capitalismo moderno a partir de la sociedad comercial (1934, 102 y 1943, 157). Para Schumpeter, la gente adinerada es similar a la burguesía rentista, que deposita su riqueza en los bancos, mientras que los banqueros son los capitalistas típicos, que otorgan créditos a los empresarios.

La interacción entre las rigideces psicológicas de los diferentes tipos de empresarios que surgen en oleadas y la naturaleza de la creación de crédito por parte del sistema bancario crea el ciclo de auge y caída. El ciclo económico schumpeteriano se compone de cuatro etapas:

En la etapa 1, los emprendedores innovadores, que utilizan el crédito creado, comienzan la producción al comprar recursos de los actores de la economía circular. Sus ideas innovadoras crean un monopolio real, utilizando el lenguaje mengeriano, y les permite cosechar ganancias monopolísticas.

En la etapa 2, los empresarios miméticos, ayudados por el crédito creado por los bancos, copian la idea innovadora y compiten por la posición de monopolio real del empresario innovador. Como consecuencia de la competencia, como en el esquema mengeriano, el nivel de ganancias se reduce a niveles cada vez más bajos. Un factor en este ciclo, propio del esquema schumpeteriano, es que los empresarios innovadores después de la innovación se convierten en meros gerentes de empresas. Al finalizar la innovación se 
restaura la economía circular, en la que la competencia provoca una disminución de los precios y una reducción de los beneficios para todos los competidores.

La etapa 3 es la fase de crisis, que comienza cuando, debido a la competencia entre empresarios en la misma esfera de actividad, las ganancias desaparecen y la producción se convierte en una actividad que genera pérdidas. En este momento, los empresarios marginales no pueden asumir el pago de intereses y esto causa quiebras, provoca una crisis de deuda y finalmente una crisis bancaria.

La etapa 4 comienza cuando al finalizar la depresión y la deflación se crean las condiciones para que una nueva generación de emprendedores innovadores compre recursos a bajo precio para iniciar una nueva ola de productos innovadores. El surgimiento de una ola de empresarios innovadores relanza el auge, esta vez a un nivel tecnológico más alto en comparación con el auge anterior.

Basándose en la idea de Wicksell, Schumpeter argumentó que el mercado de crédito bancario no está regulado por la oferta y la demanda, la oferta y la demanda no se enfrentan como fuerzas independientes: "la demanda de crédito no sólo se posibilita a sí misma, sino también a una oferta correspondiente; y cada oferta hace posible una demanda correspondiente, de modo que la oferta y la demanda en este caso no se enfrentan entre sí como fuerzas independientes" (Schumpeter 1917/1956, 207). Pero, Schumpeter no vinculó su ciclo de auge y caída con el efecto inflacionario de la creación de crédito, ni siguió la idea wickselliana del efecto perjudicial de la diferencia entre la tasa natural y la tasa de interés de los préstamos. La creación de crédito de los bancos para los empresarios no conduciría a una inflación perjudicial sostenida. Los empresarios producirían nuevas franjas de bienes, lo que equilibraría el aumento de la cantidad de dinero en la economía. En este contexto, sólo podría haber un aumento temporal de los precios, pero pronto la aparición de nuevos bienes en el mercado eliminaría la inflación, o más bien causaría la deflación (Schumpeter 1934: 110). Él solo temía el efecto inflacionario de los créditos de consumo.

Para él, el principal factor causal de los ciclos de auge y caída son los rasgos psicológicos de las diferentes clases de empresarios, y el efecto se ve multiplicado por la creación de crédito de los 
bancos, y la causa no es la diferencia entre la tasa de interés natural y la tasa de interés de los préstamos. En consecuencia, Schumpeter argumentó que ese Estado benevolente Wieseriano intervendría y, para amortiguar el impacto destructivo de los ciclos de auge y caída, regularía la expansión del crédito y aliviaría el impacto de la crisis al fomentar la expansión del crédito. Por lo tanto, Schumpeter apoyó la idea de descartar el sistema monetario basado en el oro con el fin de permitir la creación de aún más crédito para posibilitar las oportunidades de los empresarios innovadores. Característicamente, vio que el problema con John Law no era que creara medios de pago in vacuo, sino que los usaba para fines que no tuvieron éxito (Schumpeter 1939, p. 113).

Por lo tanto, aunque Schumpeter, en su modelo económico, siguió la herencia del mundo mengeriano en cuestiones clave importantes, al final recurrió a la idea de que el estado debe echar una mano al mercado que se autodesequilibra para suavizar el ciclo de desequilibrio y traer un progreso más equilibrado. El autodesequilibrio de los mercados está causado en la teoría schumpeteriana por los rígidos rasgos psicológicos inherentes en los empresarios innovadores y miméticos. Al carecer los mercados de un mecanismo para equilibrar esos rasgos psicológicos rígidos, Schumpeter llegó a una conclusión que es contraria a la idea mengeriana original: la invocación del intervencionismo estatal para suplantar el mecanismo de autocorrección del mercado.

No es de extrañar el duro rechazo de las ideas de Schumpeter por parte de Mises, quién se mantuvo fiel al programa original de Menger de defender los mercados libres sin trabas y oponerse firmemente al intervencionismo estatal. ${ }^{7}$

\footnotetext{
7 Margit Mises (1976, p. 64) registró el comentario sarcástico de Mises sobre Schumpeter: "cuando el gran profesor fue ministro de finanzas no pudo proteger a Austria contra la inflación más desastrosa de su historia y cuando el gran profesor fue presidente de un banco (Biedermann Bank), el banco quebró."
} 


\section{LA CONTINUACIÓN Y DESVIACIÓN DE LAS IDEAS MENGERIANAS EN EL TRABAJO DE MISES}

La teoría de Mises también siguió la tradición mengeriana en temas clave dentro del marco subjetivista del proceso económico. Mises defendió la no intervención del estado en la economía (Raico, 2012).

Siguió el punto de vista liberal clásico de Menger y BöhmBawerk y estaba firmemente en contra de cualquier tipo de intervencionismo estatal en la economía.

Mises construyó su edificio teórico sobre la acción humana, sobre las personas emprendedoras, como Menger. Para él, la persona que actúa, piensa, calcula y planifica, es el agente de los cambios dinámicos en la economía. Distinguió la persona que actúa (emprendedora), que es un rasgo humano inherente, del empresario, que Mises llamó "promotor" $(1949,255)$. La aventura y el afán de obtener ganancias es lo que distingue al empresario promotor de la persona que actúa. Mises, siguiendo el concepto mengeriano, añadió también una diferencia entre el uso del capital entre el promotor y la persona que actúa: es el promotor quien usa el capital: "la función del empresario no puede separarse de la dirección del empleo de factores de producción para la realización de tareas definidas" $(1949$, 302). Mises también evitó tratar al "capitalista" y al "emprendedor" como fenómenos distintos, a diferencia de Schumpeter. Argumentó que, dependiendo de las circunstancias y las elecciones personales, el capitalista podría ser diferente del empresario, pero también "las funciones del empresario, el propietario, el capitalista y el trabajador a menudo se combinan por las mismas personas". (1949, 253-4). La conceptualización del capital es similar a la de Menger (1888): "la suma del dinero equivalente de todos los activos", incluido el efectivo al mando del empresario $(1949,262)$. Mises $(1949,517)$ también argumenta que aquellos que tienen montantes de dinero como capital tienen ventaja sobre aquellos que tienen bienes de capital. El concepto de capital de Mises también es muy similar al que Menger expuso en 1883: "se denomina capital a la cifra dineraria dedicada en un momento determinado a un determinado negocio, resultante de deducir del total valor monetario del activo el total valor monetario del pasivo. 
En este orden de ideas, no tiene importancia el que los bienes así valorados sean de una u otra condición; da lo mismo que se trate de terrenos, edificios, maquinaria, herramientas, mercaderías de todo orden, créditos, efectos comerciales, metálico o cualquier otro activo" (Mises 1949, 262). Mises también argumentó que el capital no sólo se calcula en términos de dinero, sino que el capital también puede consistir en cantidades de dinero $(1949,517)$.

Pero también hay divergencias con las ideas mengerianas. Para Mises, el papel del empresario es más bien el de ajustar la producción a la demanda de los consumidores $(1949,301)$, lo que puede incluir la innovación: "la función empresarial típica consiste en determinar el empleo que deba darse a los factores de producción. El empresario es aquella persona que da a cada uno de ellos su destino específico". $(1949,288)$. Para Mises, la causa del beneficio es la anticipación empresarial $(1949,288)$, que ajusta de manera precisa la producción a la demanda de los consumidores. Esto puede incluir la ampliación del conocimiento (innovación), pero el énfasis está en la anticipación del empresario, que le permite aprovechar los bienes de capital y la inversión de una manera que anticipa la demanda del consumidor en el futuro y, por lo tanto, posibilita la obtención de ganancias para el empresario.

Para Mises, la ganancia es el beneficio derivado de la acción, rendimiento menos costes $(1949,287)$. Las pérdidas y las ganancias están completamente determinadas por el éxito o el fracaso del empresario para ajustar la producción a la demanda de los consumidores. Para él, no hay nada estático en las ganancias y nunca puede haber un equilibrio con respecto a ellas. Las pérdidas y ganancias son, por el contrario, un fenómeno de desviación de la normalidad, de cambios imprevistos por la mayoría y de un desequilibrio. (1949, 295). Las elecciones de los consumidores hacen aparecer las pérdidas y las ganancias y, por lo tanto, transfieren la propiedad de los medios de producción de manos de los menos eficientes a los más eficientes. En ausencia de pérdidas y ganancias, los empresarios no sabrían cuáles son las necesidades más urgentes de los consumidores $(1949,297)$. Claramente, no vincula el beneficio a la innovación, que crea una posición de monopolio real, sino a la capacidad del empresario de anticipar mejor que otras personas la demanda futura de los consumidores $(1949,288)$. Mientras 
que Mises también era consciente del hecho de que las ganancias estaban relacionadas con la innovación $(1949,292)$.

En su teoría del interés, Mises sigue la tradición de BöhmBawerk, y argumenta que el interés es el precio de intercambiar el bien presente por el bien futuro y así, descartó la teoría mengeriana (1949, 520-523). En el sistema misiano, el interés original es la relación entre el valor asignado a la satisfacción de deseos en el futuro inmediato y el valor asignado a la satisfacción de deseos en períodos en el futuro lejano. Se manifiesta en la economía de mercado como el descuento de bienes futuros frente a los bienes presentes. Es la tasa de interés original la que determina cuánto del suministro disponible de bienes se dedicará al consumo y cuánto se provisionará para períodos más remotos del futuro. La tasa de interés original es la compensación por la abstinencia del consumo inmediato del bien presente.

Mises apoya el concepto de interés de que la acumulación de capital está relacionada con el ahorro, con la "abstención del consumo de una parte del ingreso" $(1949,520)$, mientras que Menger descartó la teoría de Senior sobre la formación de capital basada en el ahorro, y argumentó que el ahorro proviene de la innovación y la eliminación de las ineficiencias durante el proceso de producción.

La principal contribución de Mises en la teoría monetaria es la distinción entre dinero y medios fiduciarios y el papel de la creación de crédito mediante la emisión de medios fiduciarios en los ciclos de auge y recesión en su libro de 1912 sobre la teoría del dinero y del crédito. Como explicó Mises en sus memorias, Menger y Böhm-Bawerk asumieron tácitamente la neutralidad del dinero. De este modo, no desarrollaron las ideas sobre la posible naturaleza no neutral del crédito creado por el sistema bancario, cuando hay una expansión crediticia. Sin embargo, el enfoque wickselliano ha refutado la visión mengeriana del impacto neutral de la expansión del crédito $(2009,44)$. Mises también quiso refutar las "falacias de Schumpeter" (Mises 2009, 44) que no tuvo en cuenta el impacto de la expansión del crédito en la tasa de interés, mientras descartó el impacto inflacionario de la expansión del crédito en el caso de los préstamos no destinados al consumo. Mises (1981, p.47) afirmó que el trabajo de Schumpeter puede caracterizarse por comportamientos engañosos y analogías mecanicistas. 
Para resolver el misterio de las crisis bancarias, Mises argumentó que los bancos de reserva fraccionaria están creando un nuevo tipo de medios de intercambio, que él llamó medios fiduciarios. Los medios fiduciarios tienen una apariencia externa de contar con respaldo en algún commodity, a pesar de que no hay suficientes reservas para convertir todos los medios fiduciarios en los commodities en los que se respaldan. Esto le llevó a hacer una distinción entre crédito mercancía y crédito fiduciario, este último con la apariencia de ser un crédito mercancía. Mises hizo su distinción basada en la diferencia fundamental entre billete reales y depósitos (1912, 275). Esta diferencia es la causa fundamental del fenómeno del ciclo económico, los ciclos de auge y recesión.

La adopción de la preferencia temporal de Böhm-Bawerk le permitió a Mises adoptar la idea Wickselliana de la tasa natural y conectarla con las ideas de la British Currency School (Hulsman 2007, 235). Mises sentó las bases de la TACE al hacer una distinción entre la tasa de interés original y la tasa de interés real del mercado como causa de los ciclos de auge y recesión. Los bancos fiduciarios de crédito (crédito circulante) reducen la tasa de préstamo en comparación con la tasa de interés original, mientras que los ahorradores no reducen su consumo (1912, cap. III). La menor tasa de interés da información falsa a las decisiones empresariales. Los empresarios, engañados por la reducción de la tasa de interés, calculan mal el capital disponible y participan en procesos de producción más largos y más intensivos en capital. El auge de la inversión acelera el ritmo del progreso económico y la mejora de los métodos tecnológicos. Pero, la inflación crediticia conduce a la inflación de precios de los activos y reduce el consumo de los asalariados a medida que los precios de los bienes aumentan antes y a un nivel más elevado que los salarios. El conjunto de propietarios de la población, cuya propensión a ahorrar una parte considerable de sus ingresos, sale favorecida. Mientras que los asalariados y los empleados que gastan la mayor parte de sus ingresos en consumo y ahorran poco, se ven afectados negativamente $y$, en consecuencia, deben restringir sus gastos. Por lo tanto, se está produciendo una acumulación intensificada de nuevo capital a expensas de los asalariados, los empleados y los pensionistas. Por otro lado, la inflación tarde o temprano conduce a una crisis bancaria, ya que las personas se 
dan cuenta de que la inflación provoca que sus ahorros se deprecien y si se llega a un sentimiento generalizado de tratar de adquirir cualquier cosa con valor se puede llegar a una hiperinflación. En consecuencia, la inflación no puede emplearse como una política permanente porque, cuando se continúa, da finalmente como resultado un colapso del sistema monetario $(1949,546)$

Mises también advierte que la inflación oculta el fenómeno del consumo de capital y conduce a una malinversión durante el auge artificial de la acumulación de capital debido a tres razones:

1) La inflación falsifica el cálculo económico y la contabilidad y produce el fenómeno de ganancias ilusorias. Si la depreciación anual se determina de tal manera que no se tiene en cuenta el hecho de que el reemplazo del equipo desgastado requerirá costes más altos que la cantidad por la que se compró en el pasado, obviamente se efectuará un cálculo insuficiente.

2) Si en la venta de inventarios y productos, toda la diferencia entre el precio gastado por su adquisición y el precio percibido en la venta se registra en los libros como un excedente, el error es el mismo.

3) Si el aumento de los precios de las acciones y los bienes inmuebles se considera una ganancia, la ilusión es la misma.

En consecuencia, la política inflacionista o expansionista debe dar lugar a un consumo excesivo, por un lado, y a una malinversión por el otro. Así, se desperdicia capital en aquellas inversiones no demandadas por los consumidores. El proceso inflacionario no elimina la necesidad de reajustar la producción y reasignar recursos. Simplemente lo pospone y, por lo tanto, lo hace más problemático. Mises concluye que la inflación basada en la expansión crediticia resulta en una crisis facilitada por el auge artificial de la acumulación de capital. ${ }^{8}$ La inflación tarde o temprano conduce a

${ }^{8}$ Hayek (1931/1980) amplió ese concepto de producción basada en el roundabout de Böhm-Bawerk con el enfoque Wickselliano de los ciclos económicos destacando que se produce una descoordinación entre los participantes del mercado, por lo que las 
una crisis bancaria, ya que los bancos aumentarán la tasa de interés, y este es el momento de la verdad: una tasa de interés más alta causa la interrupción de la producción y conduce a una crisis bancaria $(1949,546)$. En este mundo misiano, el efecto real de la expansión crediticia es el conocido como efecto Cantillon, es decir, aquellos que reciben primero el dinero extra (crédito) obtienen ventaja a expensas de aquellos que no lo hacen, en un juego de suma cero (North 2012, 42).

Discutiendo con Schumpeter, Mises $(1912,469)$ describe de manera reveladora el efecto de la teoría de Schumpeter comparándola con la venta de boletos en una habitación: uno puede emitir tantos boletos como desee, pero el espacio físico de la habitación sigue siendo el mismo. Mises argumenta que la dificultad fundamental es la necesidad de comparar la cantidad de dinero con el conjunto de bienes.

Contrariamente al concepto de estado benevolente de Schumpeter, Mises argumenta que la supresión de la tasa de interés es la política que "guía la política monetaria y crediticia de casi todos los gobiernos contemporáneos" (1949, 186). Las élites políticas están principalmente interesadas en suprimir la tasa de interés y crear inflación para financiar el despilfarro y el acaparamiento de poder. El intervencionismo estatal perturba el mecanismo de autocorrección de los mercados y crea un nuevo desequilibrio que, a su vez, crea la necesidad de intervenciones estatales aún más fuertes. En el esquema misiano, los bancos centrales son los actores clave en la creación de ciclos económicos a medida que controlan al sector bancario para poder seguir una estrategia de supresión de las tasas de interés.

En lo que respecta a la solución, según Salerno (2008), la posición inicial de Mises era regresar al patrón oro del 100\%, y sólo

\footnotetext{
variaciones en la tasa de interés causan distorsiones entre la cantidad producida de bienes de capital y de bienes de consumo: las tasas de interés bajas indican una mayor disposición de las personas a ahorrar (menor grado de impaciencia), lo que indica a los empresarios que inviertan en una producción más indirecta. El aumento de la tasa de interés de los bancos con el fin de atraer reservas para poder cumplir sus promesas de pago incorporadas en el dinero fiduciario, resultaría en una quiebra, ya que el aumento de los intereses perturba los planes de los empresarios marginales, cuyo negocio fue concebido bajo unos pagos dependientes de tasas de intereses más bajas.
} 
permitir la emisión de crédito mercancía por parte de los bancos. Más tarde, cambió su posición al darse cuenta de que el patrón oro del 100\% requeriría el intervencionismo estatal para obligar a los bancos a no emitir crédito fiduciario y esto le daría el poder al estado. Su posición final fue apoyar el sistema de banca libre basado en el patrón oro, sin un banco central monopolista.

III. ARGUMENTACIÓN SOBRE LA VALIDEZ DE LA T.A.C.E. A LA
LUZ DE LAS DIFERENTES TEORÍAS CONTRAPUESTAS.

Menger tenía una visión optimista sobre el funcionamiento de los mercados. Los mercados son instituciones formadas a través de la acción humana natural al tratar de lograr la mejor satisfacción posible de sus necesidades. Vio en los mercados no sólo un foro para el intercambio de bienes, sino también una institución que permite la formación de cadenas de producción entre diferentes actores con el objetivo de producir un bien de orden inferior a partir de una combinación peculiar de bienes de orden superior. Los mercados también son el lugar para que los emprendedores adquieran capital a través de préstamos, si ellos mismos no poseen el capital. El interés es el precio pagado por el uso temporal del capital por parte del emprendedor para que pueda comenzar una transformación de bienes de orden superior en bienes de orden inferior con la expectativa de obtener ganancias. Las ganancias se obtienen al alcanzar la posición de monopolio real a través de la innovación o al competir por la posición de monopolio real de un emprendedor. La competencia insta a la innovación y permite el aumento de capital a través de la innovación y la reducción de las ineficiencias.

Menger conceptualizó un mundo en desarrollo progresivo, en el que el conocimiento, las necesidades, la división del trabajo y el capital están en crecimiento, como tendencia general. El equilibrio es solo un fenómeno temporal y limitado. El desequilibrio que origina las crisis también es temporal y limitado.

En caso de crisis, los mercados están ofreciendo el espacio institucional para pensar y planificar a las personas emprendedoras 
para considerar sus planes, innovar y reinvertir su capital y capacidad de trabajo en una producción nueva e innovadora y así poder superar la crisis.

La extensión de crédito es un fenómeno benevolente en el sistema mengeriano. Menger describió un "círculo beatífico" optimista: cuanto más crédito, más personas emprendedoras podrían convertirse en empresarios. La ampliación del conocimiento empresarial aumenta el conjunto disponible de bienes y capital para el consumo y la producción futuros, y también facilita la expansión de las necesidades y, en general, el progreso de la civilización. No obstante, una de las áreas clave que Menger dejó para futuras investigaciones es el papel del sistema bancario y el impacto de la creación de crédito en la economía. Schumpeter y Mises asumieron esta tarea y llegaron a posiciones teóricas muy divergentes y conflictivas.

En cierta manera, Schumpeter siguió de cerca la tradición mengeriana en cuanto al papel de la innovación y el papel de la productividad de los empresarios interesados en crear bienes, capital, ahorros para el consumo futuro y, de hecho, expandir las necesidades humanas al inventar nuevos productos. Pero, para explicar los ciclos de auge y recesión, Schumpeter introdujo rasgos psicológicos rígidos del comportamiento humano, cuya rigidez es ajena a Menger, quien evitó la psicologización. Schumpeter, con el fin de proporcionar a las diferencias de actitud/psicología de los empresarios una fuerza creíble para el ciclo de auge y caída, introdujo el proceso de creación de crédito de la nada. Pero Schumpeter descartó el mecanismo de autorregulación del mercado en el proceso de creación de crédito. En lugar de los mercados, confiaba en la regulación benévola del estado para suavizar los ciclos de auge y recesión y disminuir sus consecuencias devastadoras. Por consiguiente, Schumpeter llegó a una posición que se enfrenta directamente con la visión mengeriana original: la invocación del intervencionismo estatal y el sistema monetario basado en el crédito estatal.

Mises combatiendo las ideas schumpeterianas también se distancia de algunas ideas mengerianas. Mises hace hincapié en que las características principales de los empresarios exitosos son la mejor anticipación de los cambios en los datos económicos, aunque 
hay referencias en sus escritos sobre la innovación y el ahorro debido al esfuerzo por una producción más eficiente por parte de los empresarios. Cambiar el énfasis a la anticipación de la innovación y la productividad de la función empresarial le permite a Mises disminuir el impacto de la productividad de la idea innovadora del capital que usa el empresario en la economía. Para Mises, el efecto de la expansión crediticia es más bien el efecto Cantillon, y no el crecimiento de la economía debido a las idea innovadoras llevadas a cabo a través de la ampliación crediticia. Una diferencia no sólo con Schumpeter, sino también con Menger. Del mismo modo, el concepto de interés misiano, que se basa en la preferencia temporal, también disminuye el papel del uso productivo esperado del capital por parte del empresario innovador. Estas modificaciones sutiles, pero importantes en el enfoque misiano en comparación con el enfoque Mengeriano, le permiten a Mises cambiar el énfasis de la ampliación del conocimiento (innovación) al cálculo, a la anticipación y al tiempo y le hace apreciar menos el impacto positivo del crédito, poniendo el énfasis en el efecto perjudicial a través de la malinversión y la inflación. Su posición está bien descrita por la metáfora que usa cuando compara la creación de crédito con la venta de boletos en una habitación, cuyas condiciones están dadas. Sin embargo, si tenemos en cuenta la teoría de Menger sobre la ampliación del conocimiento como fuente de dinamismo y también la causa de la acumulación de capital, entonces hay un alcance mucho mayor para el crecimiento debido a la ampliación del crédito que lo que permitiría la teoría de Mises: en el esquema mengeriano, mientras que los boletos se venden a los consumidores para ingresar a la sala, los empresarios están ocupados en ampliar la sala y en abrir nuevas salas para satisfacer la necesidad cada vez mayor de espacio para los clientes.

Mises, por otro lado, se mantuvo fiel al enfoque original de Menger sobre el papel del intervencionismo estatal, contrario a Schumpeter. En el marco misiano, el principal culpable de los ciclos económicos es el efecto de monopolización y cartelización del intervencionismo estatal, el secuestro del sistema monetario por parte del estado a través de la regulación gubernamental y los bancos centrales, lo que fomenta la creación de crédito fiduciario a través de la cooperación central por el control de la tasa de interés. 
La solución de Mises fue expulsar a los bancos estatales y centrales del sistema monetario, incluso una solución más radical la encontramos en la posición de Menger de un estado ideal del sistema monetario y bancario.

En cuanto a la credibilidad de la TACE, la lección principal de este análisis comparativo de los sistemas mengeriano, schumpeteriano y misiano es que si la explicación original del empresario, el beneficio, la productividad del capital y el interés de Menger es válida, cuyo marco fue seguido por Schumpeter, hasta cierto punto, entonces el sistema monetario actual tiene una latitud mucho más amplia para producir "riqueza" de lo que sugiere la TACE de Mises; que considera que el énfasis está en servir las demandas, el cálculo y la anticipación a los consumidores, y no en la innovación y productividad de la idea emprendedora.

Utilizando la teoría austriaca mengeriana, podríamos explicar que la causa del éxito del sistema monetario posterior al oro es que la expansión crediticia sin precedentes trajo consigo un auge empresarial sin precedentes. Las innovaciones empresariales y los ahorros están proporcionando más beneficios al régimen de inflación crediticia de lo que los austriacos suelen temer según la TACE de Böhm-Bawer y Mises.

Aunque no haya un colapso rápido, seguro que la hiperinflación es un peligro presente y la expansión crediticia fiduciaria siempre es, como mucho, «pan para hoy y hambre para mañana» causando distorsiones y pudiendo producir futuras crisis económicas. El crédito de nueva creación pone de entrada a disposición de los empresarios una capacidad adquisitiva que éstos gastan en proyectos de inversión desproporcionadamente ambiciosos y produce así una descoordinación generalizada en el sistema económico. La burbuja financiera, y tarde o temprano el proceso, se revierte en forma de una recesión económica en la que se inicia el doloroso y necesario reajuste que siempre exige la readaptación de toda estructura productiva real que se ha visto distorsionada por la inflación de crédito (Huerta de Soto 1998). Huerta de Soto argumenta que es esencialmente necesario reestablecer en la banca los principios legales tradicionales que regulan el contrato de depósito irregular de bienes fungibles y que requieren el mantenimiento 
continuo del tantundem; en otras palabras, un requisito de reserva del 100 por ciento para depósitos a la vista y equivalentes.

La otra lección de Mises es que el surgimiento del estado es un peligro real. La sobrerregulación, la alta carga fiscal, la cartelización de la vida económica reduce las oportunidades empresariales, lo que, a su vez, genera aún más regulación para corregir los supuestos errores de los mercados. La combinación letal para la economía es la coexistencia de la creación de crédito impulsada por el estado y la regulación estatal excesiva, lo que frena la innovación empresarial. Esta combinación puede conducir a la estanflación, e incluso al colapso económico y la hiperinflación, como en los casos de Zimbabwe o Venezuela en los últimos años. El peligro no es la hiperinflación per se debido a la expansión del crédito, sino el excesivo intervencionismo estatal en el contexto de la creación ilimitada de crédito. Tal combinación puede conducir a la hiperinflación. Tal desarrollo es posible, ya que el poder de los estados ha aumentado dramáticamente en las últimas décadas, y los estados se han convertido en el "padrino" paternalista y el regulador legítimo de la vida económica. Este aumento sin precedentes del papel del estado se debió en gran medida a la expansión crediticia centralmente marcada. El estado paternalista, basado en la ampliación de crédito, pudo corromper tanto a empresarios como a consumidores (votantes) al proporcionarles medios financieros mucho más allá de lo que permitirían los ingresos fiscales. El peligro real es la perspectiva del Zwangwirschaft del socialismo de estilo alemán (Mises, 1944), que pone en peligro la primera libertad empresarial, y la libertad personal de los ciudadanos, ya que el papel de la confianza en el estado es cada vez mayor. Una perspectiva temerosa de camino a la servidumbre (Hayek, 1944) bajo el disfraz del socialismo democrático.

\section{BIBLIOGRAFÍA}

Ahiakpor, James (2003). Classical Macroeconomics. London and New York, Routledge.

Andersen, E. S. (2009) Schumpeter's Evolutionary Economics. London and New York: Anthem Press. 
Arena R. and Gloria-Palermo S. (2001), 'Evolutionary themes in the Austrian tradition: Menger, Wieser and Schumpeter on institutions and rationality', in Garrouste P. and Ioannides S. (eds), Evolution and Path Dependence in Economic Ideas. Cheltenham, UK: Edward Elgar.

Bagehot, Walter (1885), The Postulates of English Political Economy, New York and London: Putname' Sons

Block, Wagner (2019), “I'm An 'Austrian' Economist: What Does It Mean?" https://www.lewrockwell.com/2019/03/walter-eblock/im-an-austrian-economist-what-does-it-mean/

Böhm-Bawerk, E. (1930). The Positive Theory of Capital. New York, G.E. Stechert and Co [1930].

- (1890) Capital and Interest, a critical history of economical theory. London, New York: Macmillan and Co.

- (1898). Karl Marx and the Close of His System: A Criticism. London, T.Fisher Unwin.

Braun, Eduard (2013), “Carl Menger's Contribution to Capital Theory", TUC Working Papers in Economics, No. 11.

Eichengreen, Barry and Flandreau, Marc (1985), The Gold Standard in Theory and History. London: Routledge.

Fekete, Antal (2007), “The Paradox of Interest revisited." https://professorfekete.com/articles/AEFTheParadoxOfInterestRevisited.pdf Festre', Agnes (2002), “Money, banking and dynamics: the Wicksellian Routes from Mises to Hayek and Schumpeter." Am J Econ Sociol 61:439-480

Haberler, Gottfried (1951), "Joseph Alois Schumpeter 1883-1950", in: Harris. S.E (ed). Schumpeter: Social Scientist, Harvard University Press, Cambridge

Hayek, Friedrich (1931), "A Note on the Development of the Doctrine of "Forced Saving,'” Quarterly Journal of Economics, vol. xlvii, pp. 123-133.

- (1980), Prices and Production and Other Works. Auburn, Ludwig von Mises Institute.

- (1944), The Road to Serfdom with The Intellectuals and Socialism. London, The Institute of Economic Affairs [2005].

- (1968), "Economic Thought: The Austrian school". in. Sills D.E. International Encyclopedia of Social Sciences V.4. New York MacMIllan, 458-462. 
- (1976), Introduction to Carl Menger Principles of Economics. Auburn: Ludwig von Mises Institute, 11-37.

- (1992), “Carl Menger (1840-1921).” In The Fortunes of Liberalism: Essays on Austrian Economics and the Ideal of Freedom. Vol. 4. The Collected Works of F. A. Hayek. Peter G. Klein, ed. Chicago: University of Chicago Press.

Huerta de Soto, Jesús (1998), Money, Bank Credit, and Economic Cycles, Mises Institute: Alabama [4 ${ }^{\text {th }}$ ed. 2020].

Hülsman, Guido (2007), Mises: The Last Knight of Liberalism. Auburn, Ludwig von Mises Institute.

Kirzner Israel (1990), “Commentary”. In: Hennings K, Samuels WJ (eds) Neoclassical economic theory 1870 to 1930. Kluwer Academic Publishers, Boston, Dordrecht, London, pp 242-249

McCaffrey, M. (2013), "Conflicting use of Entrepreneur in Turn-ofthe-Century Vienna". History of Economic Review (58): 27-43.

Menger, Carl (1871), Principles of Economics. Auburn: Ludwig von Mises Institute [1976].

- (1888). "Zur Theorie des Kapitals". Jahrbücher für Nationalökonomie und Statistik, 17, 1-49

- (1892), On the Origins of Money. Auburn, Ludwig von Mises institute [2009].

- (1994), Carl Menger's Lectures to Crown Prince Rudolf of Austria. Streissler, Erich and Streissler, Monika (eds). Cheltenham, Edward Elgar.

Mill, John S. (1965), Collected Works. Edited by J.M.Robson. London: University of Toronto Press [1967].

Mises, Ludwig (1912), The Theory of Money and Credit. New Heaven, Yale University Press [1953].

- (1944), Omnipotent Government: The Rise of the Total State and Total War. New Heaven: Yale University Press.

- (1949), Human Action: A Treatise on Economics, Scholar's Edition, Auburn: Ludwig von Mises Institute [1998].

- (1981), Epistemological Problems of Economics, Auburn: Ludwig von Mises Institute [3rd ed. 2003].

- (2009), Memoirs. Auburn, Ludwig von Mises Institute.

Mises, Margit (1976), My Years with Ludwig von Mises. New Rochelle, N.Y.: Arlington House. 
Raico, Raico (2012), Classical Liberalism and the Austrian School. Auburn (Alabama): Ludwig von Mises Institute.

Rothbard, Murray (1963), America's Great Depression, Mises Institute. Salerno, Joseph T. (1999a), "Carl Menger: The Founding of the Austrian School." 15 Great Austrian Economists. Holcombe, Randall G. (ed.). Auburn, Ludwig von Mises Institute.: 71-100.

- (1999b), "The place of Mises's Human Action in the development of modern economic thought." Q J Austrian Econ 2:35-65

- (2008), "Böhm-Bawerk's Vision of the Capitalist Economic Process: Intellectual Influences and Conceptual Foundations." New Perspectives on Political Economy 4(2): 87-112.

Smith, Adam (1776), An Inquiry into the Nature and Causes of the Wealth of Nations. Strand, Strahan and Cadell, Reprint: University of Oxford, Eighteenth Century Collections Online

Schumpeter, Joseph (1917), "Das Sozial Produkt und die Rechenpfennige: Glossen und Beitrage zur Geldtheorie von Heute." Archiv für Sozialwissenschaft XLIV: 627-715. Translated into English by A. Marget. "Money and the social product." International Economic Papers 6: 148-211 [1956].

- (1934), The Theory of Economic Development. New Brunswick, New Jersey, Transaction Publishers [2004].

- (1939), Business Cycles: A Theoretical, Historical and Statistical Analysis of the Capitalist Process. New York, McGraw-Hill.

- (1943), 'Capitalism in the postwar world', in S.E. Harris (ed.) Postwar Economic Problems, New York and London: McGraw-Hill.

- (1943), Capitalism, Democracy and Socialism, London: Routledge [2003]

- (1954), History of Economic Analyses, London: Routledge [1981].

Streissler Erich (1983), “Schumpeter and Hayek: On some similarities in their thought", in. Machlup-Fels-Muller-Groeling (eds.) Reflections of a Troubled World Economy, London Macmillan.

- (1987), 'Wieser F. von', in J. Eatwell M. Milate and P. Newman (eds), The New Palgrave: A dictionary of Economics (vol. 4), London: Macmillan.

Yagi, Kiichiro (1993), "Menger's Grundsitze in the Making." History of Political Economy 25 (Winter). 
Yonay, Yuval (1998), The struggle over the soul of economics: institutionalist and neoclassical economists in America between the wars. Princeton, Princeton University

Wicksell, K. (1898), Interest and Prices. New York: M. Kelley Publishers [1965].

- (1893), Value Rent and Capital. Reprinted by New York: Augustus M. Kelley Publishers [1970]

- (1907), „The Influence of the Rate of Interest on Prices” Economic Journal XVII (1907), pp. 213-220

Wieser, Friedrich (1893), Natural Value. London: MacMillan.

- (1927), Social Economics, London: Allen \& Unwin. 